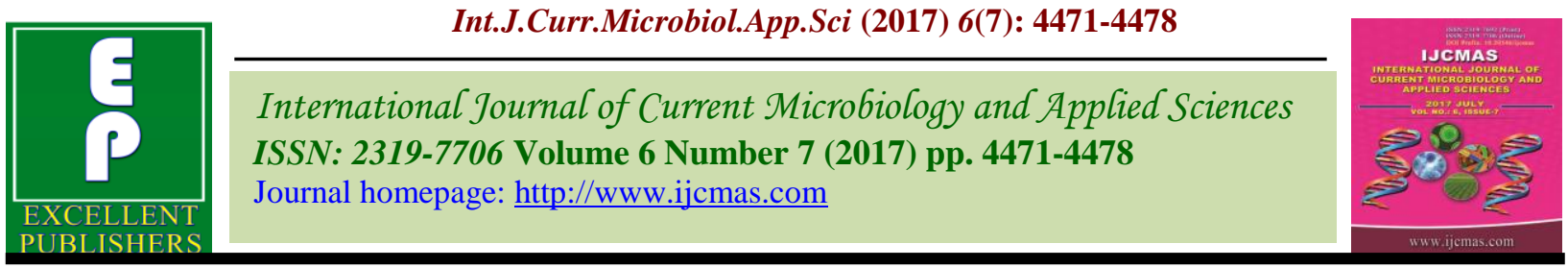

Original Research Article

https://doi.org/10.20546/ijcmas.2017.607.466

\title{
Combining Ability Studies in Bitter Gourd (Momordica charantia L.) for Quantitative Characters
}

\author{
L. Bhatt ${ }^{*}$, S.P. Singh ${ }^{1}$, A.K. Soni ${ }^{1}$ and M.K. Samota ${ }^{2}$ \\ ${ }^{1}$ Sri Karan Narendra Agriculture University, Jobner-303329, India \\ ${ }^{2}$ ICAR- Indian Agricultural Research Institute, New Delhi-110 012, India \\ *Corresponding author
}

\section{Keywords}

Genotype,

Replications,

Breeding,

Fruit harvest.

\section{Article Info}

Accepted:

xx June 2017

Available Online:

xx July 2017

\section{A B S T R A C T}

Nine diverse genotypes of bitter gourd (Momordica charantia L.) were crossed during summer season of 2015 in diallel design (excluding reciprocals).The resultant 36 hybrids along with the parents were evaluated in randomized block design with three replications during Kharif 2015 at Horticulture farm, S.K.N. College of Agriculture, Jobner. Each parents/F1 were sown in furrow maintaining $2.25 \mathrm{~m} \times 0.75 \mathrm{~m}$ spacing. The analysis of variance for experimental design revealed the existence of adequate genetic variability in experimental material for all traits under study. Variance due to parents Vs F1's were significant for all characters, except days taken to opening of first female flower,days taken to first fruit set, fruit weight and total fruits yield per vine, thereby indicating the presence of overall average heterosis for all characters. Combining ability analysis revealed importance of both additive and non-additive gene action. On the basis of overall performance the three best crosses were, namely Panipat Local x Phule Green, Phule Green x Pusa Do Mausami and Punjab-14 x Pusa Do Mausami. Panipat Local x Phule Green was superior in respect of days taken to opening first female flower, vine length, days to first fruit harvest, fruit length. Phule Green x Pusa Do Mausami was took fruit length, fruit weight, fruit diameter and total fruits yield per vine. Punjab-14 x Pusa Do Mausami was better with regard to superior for number of node at which first female flower appeared, number of fruits per vine and days to first fruit harvest. Hence could be further evaluated in future breeding programme for their large scale testing.

\section{Introduction}

Bitter gourd or balsam pear (Momordica charantia L.) is an important nutritive and commercial cucurbits belonging to the Cucurbitaceae. In terms of nutritive value, bitter gourd ranks first among cucurbits due to high value of vitamins and minerals. It is highly cross pollinated due to monoecious nature and has high degree of heterozygosity. Due to efforts of vegetable breeders, improved varieties and hybrids have been developed. Crops improvement involves strategies for enhancing yield potentiality and quality components. In bitter gourd full exploitation of heterosis through development of hybrids has not been successfully commercialized due to one or the other reason. Variability found in shape, size and colour of fruits in most conspicuous which offers tremendous scope for heterosis breeding for yield enhancement. Crossing nature and heterosis in cross pollinated crops has been known to offer good potentialities 
for increased yield. Although some information is available about combining ability, they are relevant to the specific region, genetic material involved and enviormental condition. Therefore, this study was conducted to generate information about general and specific combining ability effects for different economic traits.

\section{Materials and Methods}

The present investigation was undertaken involving different diverse genotypes/cultivars of bitter gourd were crossed in all possible combinations excluding reciprocals. The $36 \mathrm{~F} 1$ hybrids along with their nine parents were evaluated during kharif 2015 in a RBD with three replications at Horticulture Farm, S.K.N. College of Agriculture,Jobner Rajasthan. Observation were recorded on five randomly selected tagged plants from each treatment for yield and yield attributing traits viz vine Length(m), days taken to opening of first female flower, days taken to first fruits set, number of node at which first female flower appeared, male : female ratio, number of fruit per vine, days to first fruit harvest, fruit length (cm) at harvest, fruit weight $(\mathrm{g})$, fruit diameter $(\mathrm{cm})$, total fruits yield per vine $(\mathrm{kg})$ and ascorbic acid (mg)/100 (g).Combining ability were calculated in accordance with Method 2 Model 1 of Griffing (1956).

\section{Results and Discussion}

Combining ability analysis is one of the most powerful tools available which gives the estimates of mean squares due to GCA, SCA and combining ability effects and aids in selecting desirable parents and crosses for further exploitation. Analysis of variance for combining ability revealed that the genotypes showed adequate amount of variation for days taken to first female flower, days taken first fruit set, number of node to first female flower appeared, vine length at harvest, fruit weight, fruit length, fruit diameter, number fruits per vine, total fruits yield per vine and ascorbic acid (Table 1). This indicates that both additive and non-additive gene action were involved in determing these characters. Mishra et al., (1994) and Khattra et al., (2000) also reported involvement of additive and non-additive gene action days taken to first female flower, fruit weight, total fruit yield per vine. The GCA mean squares were larger in magnitude than mean SCA squares for days taken first female flower, days taken first fruit set,fruit length at harvest,fruit weight and ascorbic acid.These results are in conformity with the results of Khattra et al., (2000); Mishra et al., (1994) and Dubey and Maurya (2002). The general combining ability is primarily a function of additive and additive $x$ additive gene action. High GCA effect of a parent is a function of breeding value and hence due to additive gene action effect and /or additive $\mathrm{x}$ additive interaction effect which represents the fixable component of genetic variation (Griffting, 1956) (Tables 2 and 3 ).

Apparently, parents with good GCA effect may be presumed to possess more favourable genes for the concerned traits. In terms of better general combiner, Punjab-14 and Arka Harit were found good general combiner for earliness and vine length. Panipat Local and Phule Green were also found good general combiner for fruit weight, fruit diameter and fruit length.Punjab-14 was also found good general combiner increase the total yield per vine, number of fruits per plant whereas Punjab-14 and Panipat Local were found good general combiner for ascorbic acid content. In most of the cases, it was observed that per se performance of the parents gave a direct reflection of their respective GCA effects the parents showing highest GCA effect for a particular characters were also observed to have high mean with respect to the characters. 
Table.1 Analysis of variance for combining ability of parents and crosses for various traits in bitter gourd

\begin{tabular}{|c|c|c|c|c|c|}
\hline \multirow[t]{2}{*}{ S. No. } & \multirow[t]{2}{*}{ Characters } & \multicolumn{4}{|l|}{ Source } \\
\hline & & GCA & SCA & Error & $\sigma^{2} g / \sigma^{2} s$ \\
\hline & df & 8 & 36 & 88 & \\
\hline 1 & $\begin{array}{l}\text { Days taken to opening of first female } \\
\text { flower }\end{array}$ & $59.138 * *$ & $3.588^{*}$ & 2.227 & 3.800 \\
\hline 2 & Days taken to first fruit set & $48.532 * *$ & $3.860^{*}$ & 2.411 & 2.892 \\
\hline 3 & $\begin{array}{l}\text { Number of node at which first female } \\
\text { flower }\end{array}$ & $0.502 * *$ & $0.508^{* *}$ & 0.043 & 0.089 \\
\hline 4 & Vine length $(\mathrm{m})$ at harvest & $0.089 * *$ & $0.046 * *$ & 0.012 & 0.202 \\
\hline 5 & Male:female ratio & $49.433^{* *}$ & $8.135^{* *}$ & 1.190 & 0.631 \\
\hline 6 & Number of fruit/vine & $7.479 * *$ & $3.834 * *$ & 0.525 & 0.191 \\
\hline 7 & Days to first fruit harvest & $137.227 * *$ & $22.027 * *$ & 2.651 & 0.631 \\
\hline 8 & Fruit length $(\mathrm{cm})$ at harvest & $56.497 * *$ & $1.890 * *$ & 0.615 & 3.983 \\
\hline 9 & Fruit weight (g) & $67.454 * *$ & $6.254 * *$ & 2.830 & 1.715 \\
\hline 10 & Fruit diameter $(\mathrm{cm})$ & $0.592 * *$ & $0.201 * *$ & 0.048 & 0.322 \\
\hline 11 & Total yield per vine $(\mathrm{kg})$ & $0.022 * *$ & $0.003^{* *}$ & 0.000 & 0.848 \\
\hline 12 & Ascorbic acid $(\mathrm{mg} / 100 \mathrm{~g})$ & $0.019 * *$ & $0.002 *$ & 0.001 & 1.701 \\
\hline
\end{tabular}


Table.2 Estimates of general combining ability effect of parents for days taken to opening of first female flower, days taken to first fruit set, number of node at which first female flower appeared, vine length $(\mathrm{m})$ at harvest, male:female ratio and number of fruit/vine

\begin{tabular}{|l|l|l|l|l|l|l|l|}
\hline S.No & Parents & $\begin{array}{l}\text { Days taken to } \\
\text { opening of } \\
\text { first female } \\
\text { Flower }\end{array}$ & $\begin{array}{l}\text { Days } \\
\text { taken to } \\
\text { first fruit } \\
\text { Set }\end{array}$ & $\begin{array}{l}\text { Number of } \\
\text { node at } \\
\text { which first } \\
\text { female flower } \\
\text { appeared }\end{array}$ & $\begin{array}{l}\text { Vine } \\
\text { length } \\
\text { (m) at } \\
\text { harvest }\end{array}$ & $\begin{array}{l}\text { Male: } \\
\text { Female } \\
\text { Ratio }\end{array}$ & $\begin{array}{l}\text { Number } \\
\text { Of } \\
\text { fruit/vine }\end{array}$ \\
\hline $\mathbf{1}$ & Punjab-14 & $-2.87^{* *}$ & $-2.56^{* *}$ & $0.14^{*}$ & $0.08^{*}$ & $-3.89^{* *}$ & $1.77^{* *}$ \\
\hline $\mathbf{2}$ & Arka Harit & $-2.14^{* *}$ & $-1.83^{* *}$ & $-0.31^{* *}$ & $-0.09^{* *}$ & $3.15^{* *}$ & 0.40 \\
\hline $\mathbf{3}$ & Panipat Local & 0.13 & -0.19 & $0.14^{*}$ & $0.08^{*}$ & $2.33^{* *}$ & $-0.60^{* *}$ \\
\hline $\mathbf{4}$ & Phule Green & $2.86^{* *}$ & $2.72^{* *}$ & $0.23^{* *}$ & $0.10^{* *}$ & $0.75^{*}$ & 0.13 \\
\hline $\mathbf{5}$ & Pusa Vishesh & $2.68^{* *}$ & $2.44^{* *}$ & -0.04 & $0.08^{*}$ & $-1.41^{* *}$ & $-1.05^{* *}$ \\
\hline $\mathbf{6}$ & Pusa Do Mausami & $2.68^{* *}$ & $2.54^{* *}$ & $0.23^{* *}$ & 0.00 & $0.88^{* *}$ & $-0.69^{* *}$ \\
\hline $\mathbf{7}$ & Kalyanpur Barahmasi & 0.50 & 0.17 & 0.05 & 0.00 & -0.10 & 0.31 \\
\hline $\mathbf{8}$ & Kalyanpur Sona & $-2.14^{* *}$ & $-1.92^{* *}$ & $-0.13^{*}$ & $-0.12^{* *}$ & $-1.42^{* *}$ & 0.04 \\
\hline $\mathbf{9}$ & Pant Karela-1 & $-1.69^{* *}$ & $-1.37^{* *}$ & $-0.31^{* *}$ & $-0.12^{* *}$ & -0.30 \\
\hline & Standard error (SEd+) & & & & & -0.32 \\
\hline & Gi & Gi-Gj & 1.467 & 1.526 & 0.205 & 0.107 & 1.073 \\
\hline
\end{tabular}

*,** Significant at $5 \%$ and $1 \%$ level, respectively 
Table.3 Estimates of general combining ability effect of parents for days to first fruit harvest, fruit length (cm) at harvest. fruit weight $(\mathrm{g})$, fruit diameter $(\mathrm{cm})$, total yield per vine $(\mathrm{kg})$ and ascorbic acid $(\mathrm{mg} / 100 \mathrm{~g})$

\begin{tabular}{|c|c|c|c|c|c|c|c|}
\hline S.No & Parents & Days to first harvest & $\begin{array}{c}\text { Fruit length } \\
(\mathrm{cm}) \text { at harvest }\end{array}$ & Fruit weight (g) & $\begin{array}{c}\text { Fruit diameter } \\
(\mathrm{cm})\end{array}$ & $\begin{array}{l}\text { Toatal yield per } \\
\text { vine (kg) }\end{array}$ & $\begin{array}{c}\text { Ascorbic } \\
\text { acid } \\
(\mathbf{m g} / 100 g)\end{array}$ \\
\hline 1 & Punjab-14 & 0.30 & $-4.17 * *$ & $-3.21 * *$ & $-0.39 * *$ & $0.07 * *$ & $0.06^{* *}$ \\
\hline 2 & Arka Harit & $-3.24 * *$ & $-2.10 * *$ & $-1.03 *$ & 0.12 & $0.04 * *$ & 0.02 \\
\hline 3 & Panipat Local & $5.12 * *$ & $1.12^{* *}$ & $2.61 * *$ & $0.44 * *$ & $0.03 * *$ & $0.06^{* *}$ \\
\hline 4 & Phule Green & $4.58 * *$ & $3.38 * *$ & $2.97 * *$ & 0.12 & 0.01 & 0.01 \\
\hline 5 & Pusa Vishesh & -0.42 & $-0.90 * *$ & -0.85 & $-0.17 * *$ & $-0.07 * *$ & 0.00 \\
\hline 6 & Pusa Do Mausami & $-4.97 * *$ & $2.05^{* *}$ & $3.70^{* *}$ & 0.01 & 0.01 & -0.02 \\
\hline 7 & Kalyanpur Barahmasi & $2.76 * *$ & $0.96^{* *}$ & $-1.03 *$ & -0.06 & $-0.04 * *$ & $-0.05 * *$ \\
\hline 8 & Kalyanpur Sona & $-1.33 * *$ & $-0.75 * *$ & $-2.48 * *$ & -0.12 & $-0.05^{* *}$ & $-0.04 * *$ \\
\hline \multirow[t]{4}{*}{9} & Pant Karela-1 & $-2.79 * *$ & 0.41 & -0.67 & 0.06 & -0.01 & $-0.05 * *$ \\
\hline & Standard error $(\mathrm{SEd}+)$ & & & & & & \\
\hline & Gi & 1.067 & 0.514 & 1.102 & 0143 & 0.017 & 0.022 \\
\hline & $\mathrm{Gi}-\mathrm{Gj}$ & 1.601 & 0.771 & 1.654 & 0.215 & 0.026 & 0.034 \\
\hline
\end{tabular}


Table.4 Estimates of specific combing ability effect of crosses for days taken to opening of first female flower, days taken to first fruit set, number of node at which first female flower appeared, vine length (m) at harvest, male:female ratio and number of fruit/vine

\begin{tabular}{|c|c|c|c|c|c|c|c|}
\hline S.No & Genotype & $\begin{array}{c}\text { Days taken } \\
\text { to opening } \\
\text { of first } \\
\text { female } \\
\text { flower }\end{array}$ & $\begin{array}{c}\text { Days } \\
\text { Taken } \\
\text { to first } \\
\text { Fruit } \\
\text { Set }\end{array}$ & $\begin{array}{c}\text { Number } \\
\text { of node } \\
\text { at which } \\
\text { first } \\
\text { female } \\
\text { flower } \\
\text { appeared }\end{array}$ & $\begin{array}{c}\text { Vine } \\
\text { length } \\
\text { (m) at } \\
\text { harvest }\end{array}$ & $\begin{array}{l}\text { Male: } \\
\text { female } \\
\text { ratio }\end{array}$ & $\begin{array}{c}\text { Number } \\
\text { Of } \\
\text { fruits/vine }\end{array}$ \\
\hline 1 & Punjab-14 x Arka Harit & -2.75 & -2.13 & -0.07 & $0.45 * *$ & $-2.56^{*}$ & 0.67 \\
\hline 2 & Punjab-14 x Panipat Local & -0.02 & -0.76 & $-0.53^{* *}$ & -0.11 & $-3.62^{* *}$ & $1.67^{*}$ \\
\hline 3 & Punjab-14 x Phule Green & 1.25 & 2.33 & $1.38 \% *$ & 0.07 & -0.53 & -1.05 \\
\hline 4 & Punjab-14 x Pusa Vishesh & 0.44 & 0.60 & -0.35 & -0.11 & 0.39 & $3.13 * *$ \\
\hline 5 & Punjab-14 x Pusa Do Mausami & 1.44 & 0.51 & 0.38 & $-0.24 *$ & -1.19 & $2.76^{* * *}$ \\
\hline$\frac{\pi}{6}$ & Punjab-14 x Kalyanpur Barahmasi & 1.62 & 1.87 & $-1.44 * *$ & $-0.64 * *$ & 1.97 & -0.24 \\
\hline 7 & Punjab-14 x Kalyanpur Sona & 0.25 & 0.96 & -0.25 & -0.11 & 1.05 & -0.96 \\
\hline$\frac{1}{8}$ & Punjab-14 x Pant Karela-1 & $-4.20^{* * *}$ & $-4.58^{* * *}$ & -0.07 & 0.09 & 0.82 & -0.60 \\
\hline 9 & Arka Harit x Panipat Local & -0.75 & -0.49 & -0.07 & -0.15 & -0.26 & 0.04 \\
\hline 10 & Arka Harit x Phule Green & -0.48 & -1.40 & -0.16 & 0.04 & $4.80^{* *}$ & 1.31 \\
\hline 11 & Arka Harit x Pusa Vishesh & -0.29 & -0.13 & $-0.89^{* *}$ & $-0.35^{* *}$ & -1.80 & 0.49 \\
\hline 12 & Arka Harit x Pusa Do Mausami & 0.71 & -0.22 & -0.16 & -0.07 & $3.41 * *$ & 1.13 \\
\hline 13 & Arka Harit x Kalyanpur Barahmasi & -1.11 & 0.15 & 0.02 & -0.07 & 1.35 & -0.87 \\
\hline 14 & Arka Harit x Kalyanpur Sona & 1.53 & 2.24 & $1.20 * *$ & $0.26^{*}$ & 0.42 & 0.40 \\
\hline 15 & Arka Harit x Pant Karela-1 & 0.07 & -0.31 & $-0.62 * *$ & -0.15 & $3.45^{* * *}$ & -1.24 \\
\hline 16 & Panipat Local x Phule Green & 0.25 & -0.04 & 0.38 & 0.07 & $-2.32^{*}$ & $-1.69^{*}$ \\
\hline 17 & Panipat Local x Pusa Vishesh & $-3.56^{*}$ & -2.76 & -0.35 & -0.11 & $3.87 * *$ & $2.49 * *$ \\
\hline 18 & Panipat Local x Pusa Do Mausami & -1.56 & -1.85 & 0.38 & 0.16 & 1.38 & -0.87 \\
\hline 19 & Panipat Local x Kalyanpur Barahmasi & -0.38 & -0.49 & $-0.44 *$ & -0.04 & $4.16^{* *}$ & 0.13 \\
\hline 20 & Panipat Local x Kalyanpur Sona & 1.25 & 1.60 & -0.25 & -0.11 & 0.70 & $-1.60^{*}$ \\
\hline 21 & Panipat Local x Pant Karela-1 & 0.80 & 1.05 & -0.07 & 0.09 & $3.02^{* * *}$ & $1.76^{*}$ \\
\hline 22 & Phule Green x Pusa Vishesh & -0.29 & -0.67 & $0.56^{* *}$ & $0.27 *$ & -1.64 & -0.24 \\
\hline 23 & Phule Green x Pusa Do Mausami & -2.29 & -0.76 & $-0.71 * *$ & -0.05 & -1.31 & -0.60 \\
\hline 24 & Phule Green x Kalyanpur Barahmasi & 1.89 & 1.60 & $-0.53^{* *}$ & -0.05 & 1.16 & 0.40 \\
\hline$\frac{25}{25}$ & Phule Green x Kalyanpur Sona & 1.53 & 1.69 & -0.35 & -0.13 & 0.70 & $1.67 *$ \\
\hline 26 & Phule Green x Pant Karela-1 & -0.93 & -0.85 & $-1.16^{* *}$ & $-0.33^{* * *}$ & $4.75 * *$ & -0.96 \\
\hline 27 & Pusa Vishesh x Pusa Do Mausami & 1.89 & 1.51 & $0.56^{* *}$ & 0.16 & $-3.28^{* *}$ & $-1.42^{*}$ \\
\hline 28 & Pusa Vishesh x Kalyanpur Barahmasi & 0.07 & -0.13 & -0.25 & -0.04 & -0.53 & $-3.42^{* *}$ \\
\hline 29 & Pusa Vishesh x Kalyanpur Sona & 1.71 & 1.96 & $0.93 * *$ & 0.09 & 0.81 & $-3.15 * *$ \\
\hline 30 & Pusa Vishesh x Pant Karela-1 & 0.25 & 0.42 & 0.11 & -0.11 & 0.40 & 0.22 \\
\hline 31 & $\begin{array}{l}\text { Pusa Do Mausami x Kalyanpur } \\
\text { Barahmasi }\end{array}$ & 0.07 & -0.22 & $0.47 *$ & $0.24 *$ & -1.38 & $-1.78 *$ \\
\hline 32 & Pusa Do Mausami x Kalyanpur Sona & 0.71 & 0.87 & -0.35 & -0.04 & $4.86^{* *}$ & $-1.51^{*}$ \\
\hline 33 & Pusa Do Mausami x Pant Karela-1 & -0.75 & -0.67 & $-1.16^{* * *}$ & $-0.24^{*}$ & 1.03 & $1.85^{* *}$ \\
\hline 34 & $\begin{array}{l}\text { Kalyanpur Barahmasi } \mathrm{x} \text { Kalyanpur } \\
\text { Sona }\end{array}$ & -1.11 & -0.76 & -0.16 & -0.04 & $-1,32$ & $3,49 * *$ \\
\hline 35 & Kalyanpur Barahmasi x Pant Karela-1 & 0.44 & -0.31 & $1.02^{* * *}$ & 0.16 & 0.14 & $1.85 \% *$ \\
\hline \multirow[t]{6}{*}{36} & Kalyanpur Sona x Pant Karela-1 & $-4.93^{* *}$ & $-5.22 * *$ & -0.80 & -0.11 & $-3.34 * *$ & $4.13 * *$ \\
\hline & Standard error & & & & & & \\
\hline & Sii & 2.451 & 2.550 & 0.342 & 0.180 & 1.792 & 1.190 \\
\hline & $\mathrm{Sij}$ & 2.771 & 2.882 & 0.387 & 0.203 & 2.026 & 1.346 \\
\hline & Sij-ik & 4.085 & 4.250 & 0.570 & 0.300 & 2.987 & 1.984 \\
\hline & Sij-Skl & 3.876 & 4.032 & 0.41 & 0.285 & 2.834 & 1.883 \\
\hline
\end{tabular}


Table.5 SCA effect for days to first fruit harvest, fruit length $(\mathrm{cm})$ at harvest, fruit weight $(\mathrm{g})$, fruit diameter $(\mathrm{cm})$, total fruits yield per vine $(\mathrm{kg})$ and ascorbic acid $(\mathrm{mg} / 100 \mathrm{~g})$

\begin{tabular}{|c|c|c|c|c|c|c|c|}
\hline S.No & Genotype & $\begin{array}{l}\text { Days to first } \\
\text { fruit harvest }\end{array}$ & $\begin{array}{c}\text { Fruit } \\
\text { Length } \\
\text { (cm) at Harvest }\end{array}$ & $\begin{array}{c}\text { Fruit } \\
\text { weight }(g)\end{array}$ & $\begin{array}{c}\text { Fruit } \\
\text { diameter } \\
(\mathrm{cm})\end{array}$ & $\begin{array}{c}\text { Total fruits } \\
\text { yield per } \\
\text { vine }(\mathrm{kg}) \\
\end{array}$ & $\begin{array}{c}\text { Ascorbic } \\
\text { acid (mg/ } \\
100 \mathrm{~g}) \\
\end{array}$ \\
\hline 1 & Punjab-14 x Arka Harit & 1.87 & $2.08 * *$ & 2.51 & 0.28 & $0.13 * *$ & 0.00 \\
\hline 2 & Punjab-14 x Panipat Local & $-3.49^{*}$ & $1.74 *$ & 0.87 & 0.36 & $-0.07 *$ & $-0.07 *$ \\
\hline 3 & Punjab-14 x Phule Green & $-5.95 * *$ & $-2.79 * *$ & $-7.49 * *$ & $-1.12 * *$ & -0.05 & 0.03 \\
\hline 4 & Punjab-14 x Pusa Vishesh & $3.05^{*}$ & 0.68 & 0.33 & 0.37 & -0.01 & 0.01 \\
\hline 5 & Punjab-14 x Pusa Do Mausami & $5.60 * *$ & $-1.67 *$ & -2.22 & $-0.81^{* *}$ & 0.00 & 0.05 \\
\hline 6 & $\begin{array}{l}\text { Punjab-14 x Kalyanpur } \\
\text { Barahmasi }\end{array}$ & $-4.13 * *$ & -0.37 & 0.51 & $-0.73 * *$ & $0.08 * *$ & 0.04 \\
\hline 7 & Punjab-14 x Kalyanpur Sona & 0.96 & 1.33 & $3.96 *$ & -0.28 & 0.02 & 0.03 \\
\hline 8 & Punjab-14 x Pant Karela-1 & 1.42 & -0.63 & $-3.85^{*}$ & -0.06 & $-0.06^{*}$ & 0.06 \\
\hline 9 & Arka Harit x Panipat Local & $-7.95 * *$ & -1.41 & 0.69 & 0.25 & $0.09 * *$ & -0.05 \\
\hline 10 & Arka Harit x Phule Green & $-5.40 * *$ & $-1.87^{*}$ & 0.33 & $-0.43^{*}$ & $-0.05^{*}$ & -0.01 \\
\hline 11 & Arka Harit x Pusa Vishesh & -0.40 & -0.19 & 0.15 & 0.27 & $0.07 *$ & -0.01 \\
\hline 12 & Arka Harit x Pusa Do Mausami & $3.15^{*}$ & -0.14 & 1.60 & -0.12 & $-0.09 * *$ & 0.04 \\
\hline 13 & $\begin{array}{l}\text { Arka Harit x Kalyanpur } \\
\text { Barahmasi }\end{array}$ & $-3.58 *$ & 0.35 & $-3.67 *$ & -0.24 & $-0.08 * *$ & 0.02 \\
\hline 14 & Arka Harit x Kalyanpur Sona & 1.51 & -0.54 & $-4.22 * *$ & -0.19 & $-0.07 * *$ & 0.03 \\
\hline 15 & Arka Harit x Pant Karela-1 & 1.96 & -1.10 & -2.04 & -0.17 & $-0.07 * *$ & 0.06 \\
\hline 16 & Panipat Local x Phule Green & -0.76 & -0.88 & -1.31 & -0.35 & -0.05 & 0.00 \\
\hline 17 & Panipat Local x Pusa Vishesh & $4.24 * *$ & -0.81 & -1.49 & -0.26 & -0.05 & -0.01 \\
\hline 18 & $\begin{array}{l}\text { Panipat Local x Pusa Do } \\
\text { Mausami }\end{array}$ & $6.78 * *$ & 0.64 & -0.04 & 0.16 & 0.00 & 0.05 \\
\hline 19 & $\begin{array}{l}\text { Panipat Local x Kalyanpur } \\
\text { Barahmasi }\end{array}$ & -2.95 & 1.13 & 0.69 & -0.17 & -0.04 & 0.06 \\
\hline 20 & Panipat Local x Kalyanpur Sona & $4.15^{* *}$ & -0.76 & -1.85 & -0.32 & -0.02 & 0.05 \\
\hline 21 & Panipat Local x Pant Karela-1 & $4.60 * *$ & $1.88 *$ & 0.33 & -0.30 & 0.02 & $0.08^{*}$ \\
\hline 22 & Phule Green x Pusa Vishesh & $3.78^{*}$ & -0.67 & 0.15 & 0.27 & $0.09 * * *$ & -0.02 \\
\hline 23 & $\begin{array}{l}\text { Phule Green x Pusa Do } \\
\text { Mausami }\end{array}$ & $4.33 * *$ & -0.21 & -0.40 & 0.28 & $0.06^{*}$ & -0.01 \\
\hline 24 & $\begin{array}{l}\text { Phule Green x Kalyanpur } \\
\text { Barahmasi }\end{array}$ & 0.60 & -1.12 & -1.67 & -0.24 & -0.02 & 0.05 \\
\hline 25 & Phule Green x Kalyanpur Sona & 1.69 & -1.01 & -0.22 & 0.01 & 0.00 & 0.00 \\
\hline 26 & Phule Green x Pant Karela-1 & $4.15 * *$ & -0.37 & 1.96 & -0.37 & 0.00 & 0.04 \\
\hline 27 & $\begin{array}{l}\text { Pusa Vishesh x Pusa Do } \\
\text { Mausami }\end{array}$ & 2.33 & -0.94 & 1.42 & -0.03 & 0.02 & 0.05 \\
\hline 28 & $\begin{array}{l}\text { Pusa Vishesh x Kalyanpur } \\
\text { Barahmasi }\end{array}$ & $-4.40 * *$ & -0.25 & 0.15 & -0.15 & 0.02 & 0.01 \\
\hline 29 & Pusa Vishesh x Kalyanpur Sona & $-4.31 * *$ & 0.86 & -0.40 & -0.30 & -0.04 & 0.02 \\
\hline 30 & Pusa Vishesh x Pant Karela-1 & -1.85 & 0.10 & -0.22 & -0.08 & -0.04 & 0.02 \\
\hline 31 & $\begin{array}{l}\text { Pusa Do Mausami x Kalyanpur } \\
\text { Barahmasi }\end{array}$ & $-6.85^{* *}$ & 0.61 & -0.40 & 0.27 & 0.03 & -0.02 \\
\hline 32 & $\begin{array}{l}\text { Pusa Do Mausami x Kalyanpur } \\
\text { Sona }\end{array}$ & -6.76 & -0.08 & 3.05 & -0.08 & 0.00 & -0.01 \\
\hline 33 & Pusa Do Mausami x Pant Karela-1 & $-4.31 * *$ & 0.75 & -0.76 & -0.06 & 0.01 & -0.04 \\
\hline 34 & $\begin{array}{l}\text { Kalyanpur Barahmasi x } \\
\text { Kalyanpur Sona }\end{array}$ & $7.51 * *$ & 0.61 & 1.78 & $0.79 * *$ & 0.05 & -0.03 \\
\hline 35 & $\begin{array}{l}\text { Kalyanpur Barahmasi x Pant } \\
\text { Karela-1 }\end{array}$ & $8.96 * *$ & 0.44 & 1.96 & 0.21 & -0.03 & -0.05 \\
\hline 36 & Kalyanpur Sona x Pant Karela-1 & 0.05 & 0.15 & $3.42 *$ & 0.27 & 0.03 & 0.02 \\
\hline & Standard error & & & & & & \\
\hline & Sii & 2.674 & 1.288 & 2.763 & 0.360 & 0.044 & 0.057 \\
\hline & $\mathrm{Sij}$ & 3.023 & 1.456 & 3.123 & 0.407 & 0.049 & 0.064 \\
\hline & Sij-ik & 4.457 & 1.796 & 4.605 & 0.600 & 0.073 & 0.095 \\
\hline & Sij-Skl & 4.229 & 2.146 & 4.368 & 0.569 & 0.069 & 0.090 \\
\hline
\end{tabular}

The results are in conformity with the finding of Munshi and Sirohi (1994), Munshi and Verma (1999) in muskmelon and Tiwari et al., (2001) in bitter gourd. The crosses having high desirable SCA effects for the economically important characters were Kalyanpur Sona $\mathrm{x}$ Pant Karela-1 and Punjab-14 x Pant Karela-1 for days taken to opening of first female flower, Kalyanpur Sona x Pant Karela-1 and Punjab-14 $\mathrm{x}$ Pant Karela-1 days taken to first fruit set, 
Punjab-14 x Phule Green and Kalyanpur Barahamasi x Pant Karela-1 for number of node at which female flower appeared, Punjab-14 x Arka Harit for vine length, Pusa Do Mausami xKalyanpur Sona and Arka Harit x Phule Green for male : female ratio, Kalyanpur Sona x Pant Karela-1 and Kalyanpur Barahamasi x Pant Karela-1 for number of fruits per plant, Arka Harit x Panipat Local, Pusa Do Mausami xKalyanpur Barahamasi for days to first fruit harvest, Punjab-14 x Arka Harit, Punjab-14 x Panipat Local for fruit length, Punjab-14 x Kalyanpur Sona for fruit weight, Kalyanpur Barahamasi $\mathrm{x}$ Kalyanpur Sona for fruit diameter, Punjab-14 x Arka Harit, Phule Green $x$ Pusa Do Mausami for total fruits yield per plant, Panipat Local $\mathrm{x}$ Pant Karela-1 for ascorbic acid content. Another important fact worth mentioning here is that the above mentioned hybrids along with possessing high SCA effects, also exhibited considerable amount of heterosis over their better parents for economically important character like earliness, number of fruits per plant (Tables 4 and 5). The mean performance of the hybrids showed more or less parallel trend with SCA effects for the above mentioned character. The hybrids which gave good per se performance also mentioned characters. The hybrids which gave good per se performance also expressed favourable high SCA effects. This explained that per se performance of hybrids was reflected in their respective SCA effects. It is noteworthy that the crosses which manifested greater SCA effects in desirable direction also exhibited favourable high heterosis over better parent. Hence, the mean performance of hybrid could be envisaged as a criteria of SCA effects and selection of crosses based on per se performance would be realistic. The present finding corroborated the earlier work of Sharma et al., (1993) in bottle gourd, Kumar and Singh (1997) in bottle gourd and Khattra et al., (2000) in bitter gourd.

\section{References}

Dubey, S. K. and Maurya, I. B. 2002. Heterosis and combining ability in bittergourd. In: International conference on vegetable, November, 11-14 Banglore, pp.-4.

Griffing, J. B. 1956. Concept of general and specific ability in relation to diallel crossing system. Australia Journal of Bio Science 9: 463-493.

Mishra, H. N Mishra, R. S, Mishra, S. N. and Parhi, G. 1994. Heterosis and combining ability in bitter gourd (Momordica charantia L.). Indian Journal of Agriculture, 64:310-313.

Munshi, A. D. and Sirohi, P. S.1994. Combining ability estimates in bitter gourd (Momordica charantia L.) Vegetable Science 21:132-136

Munshi, A. D. and Verma, V. K. 1999. Combining ability in muskmelon (Cucumis melo). Indian Journal Agriculture, 69:210-220

Khattra, A. S, Singh, N. J. and Thakar, J. C. 2000. Studies on combining ability in bitter gourd. Vegetable Science.21:158162.

Kumar, S., Singh, W. K. 1997. Line x tester analysis for combining ability and heterosis in bottle gourd (Lagenaria siceraria (Mol.) Standl.). Vegetable Science 25 (1):78-80.

Sharma, N. K., Dhankar, B. A and Tewatia, A. S. 1993. Heterosis in bottlegourd (Lagenaria sicenaria (Mol). Stand). Haryana Agriculture University Journal of Research. 23:8-14.

Tiwari, D., Ram, H.H. and Jaiswal. H.R. 2001. Studies on heterosis and combining ability in indigenous bitter gourd (Momordica charantia L.) for fruit yield. Vegetable Science 28(2):106-108.

\section{How to cite this article:}

Bhatt, L., Singh, S.P., Soni, A.K. and Samota, M.K. 2017. Combining Ability Studies in Bitter Gourd (Momordica charantia L.) for Quantitative Characters. Int.J.Curr.Microbiol.App.Sci. 6(7): 4471-4478. doi: https://doi.org/10.20546/ijcmas.2017.607.466 